\section{8 \\ MORE DISCHARGES, LESS FOLLOW-UP AND SIMILAR RATES OF CORONARY ANGIOGRAPHY: INITIAL 'REAL-WORLD' EXPERIENCE OF NICE GUIDANCE ON ASSESSMENT OF CHEST PAIN OF RECENT ONSET IN THE RAPID ACCESS CHEST PAIN CLINIC}

C Sheppard, J Edmund, K Frawley, G Dubey, J Baron, S Burn, TAzeem, M Bhandari, K Chitkara, A Tukan, A McCance, D J Kelly Royal Derby Hospital

\section{doi:10.1136/heartjnl-2013-304019.128}

Introduction In March 2010 NICE clinical guideline number 95 described a new protocol-driven pathway for the investigation of chest pain of recent onset. This emphasised the importance of establishing pre-test probability of coronary artery disease and encouraged the use of non-invasive coronary and myocardial perfusion imaging. We sought to determine the impact of these changes on resource utilisation in particular coronary angiography and need for out-patient review.

Methods Clinical disposition for patients attending the nurse-led Rapid access chest pain clinic (RACPC) at Royal Derby hospital between January and August 2011 was analysed using a dedicated database. During this period an established exercise tolerance test-driven protocol was in place. The proposed NICE RACPC protocol was applied retrospectively to this cohort to produce a projected model of outcomes. The new RACPC protocol was implemented in July 2012 and disposition was recorded prospectively for 'real-world' patients attending between July and November 2012. Proportions were compared with Pearson's $\chi^{2}$ test and $p$ value of $<0.05$ was taken to be significant.

Results Results are summarised in figure 1. A total of 916 patients were analysed: 360 patients attended RACPC between July and November 2012 and 556 during 2011. Of these 517 (56.4\%) were male. 364 (39.7\%) were deemed inappropriate referrals by NICE criteria and were discharged to the GP. Typical angina was diagnosed in 191 (20.8\%) and non-angina symptoms in 333 (36.4\%). 562 (61.4\%) underwent a treadmill exercise tolerance test, of which 136 (14.9\%) were reported as positive for ischaemia.

Implementation of the new protocol-driven RACPC pathway during 2012 resulted in a significant reduction in cardiology clinic follow-up compared with 2011 ( $2 \%$ vs $23 \%$, p<0.0001). Rates of discharge to the GP upon initial consultation were higher $(67.5 \%$ vs $60 \%, p=0.02)$ with fewer direct admissions from RACPC $(3 \%$ vs $6 \%, p=0.008)$, whereas the rate of coronary angiography $(10.3 \%$ vs $8 \%, p=0.57)$ was similar. The rate of myocardial perfusion scanning remained similar $(9.2 \%$ vs $6 \%, p=0.13)$ while uptake of CT coronary angiography currently was lower than predicted by NICE at $2.5 \%$. Observed rates of angiography and MPS during 2012 were similar to those predicted by the NICE retrospective model.

Conclusions In this 'real-world' study, implementation of the 2010 NICE guidance on the initial evaluation of chest pain of recent onset with a protocol-driven RACPC pathway resulted in a greater proportion of initial discharge to primary care with less frequent outpatient review and similar rates of referral for coronary angiography. Utilisation of non-invasive coronary imaging in the initial assessment of chest pain is likely to expand and longitudinal outcome studies are required to confirm the safety of revised RACPC protocols.

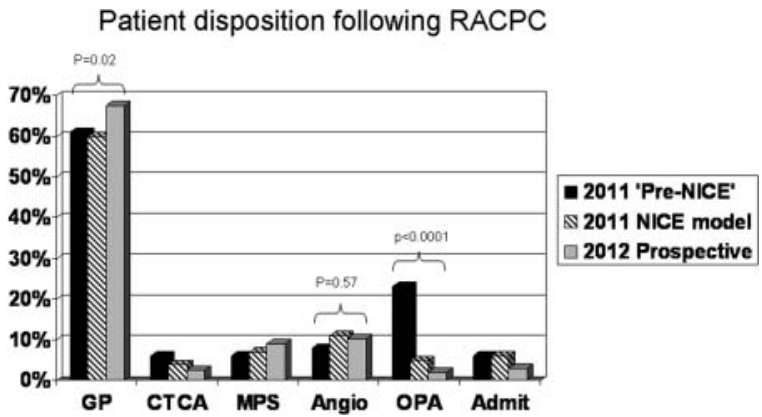

Figure 1 Patient dispositions following RACPC attendance (A) during 2011 ( $n=556)$ under the existing RACPC protocol (B) using retrospective modelling of the proposed new NICE-recommended protocol applied to the same cohort of patients, (C) July to November 2012 ( $n=360$ ) using the revised NICE protocol. Legend: GP- discharged to general practice; CTCA- CT coronary angiogram; MPS- stress myocardial perfusion scan; Angio- Coronary angiography; OPA- referred for cardiology review without further tests; Admit- Direct admission to hospital from RACPC. 